\section{Framing the Subject : a subject indexing model for electronic bibliographic databases in the humanities}

\section{Hazel Rothera}

\section{The Author}

Hazel Rothera graduated from Cambridge University in 1994 with a first class BA in Spanish and French. After a year working in Trinity College (Cambridge) Library she went to the University of Central England in Birmingham to take an MA in Information and Library Studies. She then moved to Paris where she is about to complete a two year contract with the British Council - after which she plans to return to the UK.

\section{The Occasion}

The dissertation on which this article is based was, in 1997, adjudged the winner from a strong entry for the 1996 LIRG Postgraduate Prize. The prize was presented in Paris by Ros Cotton, on behalf of the Group, in June 1998.

\section{Abstract}

This article reviews in detail an MA dissertation to assess the scope and value of electronic bibliographic databases in the humanities. A model is developed and demonstrated to determine essential and desirable indexing terms and to highlight some inherent complexities. Features of commercially available databases are assessed against this model. The author concludes with some personal observations on the dissertation experience and on prospects for further research in this area.

\section{Acknowledgements}

Thanks are due to the staff and students on the MA course at UCE; particularly to my supervisor, Bruce Reid, and also to the MA course tutors David Butcher and Michael Shoolbred. Also to the library staff at UCE, to my colleagues at the British Council Library in Paris, and to my parents - whose support, both moral and financial, was essential.

\section{Background to research}

The research for Framing the Subject, my dissertation for the MA in Information and Library Studies at the University of Central England, was carried out over the summer of 1996. Time constraints were tight, and in the event, final editing and proof-reading had to be fitted around settling into a new country and job! The topic of the research first suggested itself in early 1996 on the MA course. As I became familiar with a variety of bibliographic databases, it quickly became apparent that those covering humanities disciplines often had much less thorough subject indexing than scientificallyoriented databases, and were correspondingly much harder to gain useful results from.

I became interested in investigating the problem further, and in particular in devising a quantitative method of testing the thoroughness of subject indexing. I was also interested, as an ex-student of European languages and literature, in applying my own academic perspective to devising more effective forms of subject indexing.

From a purely practical point of view, this research topic had further advantages for a fulltime MA student. A valid methodology could be devised based largely on my own analysis and consideration of readily available data. This would avoid the difficulties of some fellow students attempting more "people-based" research - who needed the cooperation of subjects to be interviewed, to form focus groups, or to fill in questionnaires 'for yet another Masters student'! It made the data collection element of the dissertation much less time-consuming and less stressful.

\section{Aims and Methodology}

The aims and methodology eventually formulated for the research project were "to devise an indexing framework for bibliographic databases which would enable effective subject retrieval in the humanities". 
The methodology was

- Review existing research into the information needs of humanists and indexing of humanities databases, using this to formulate criteria for the subject indexing of humanities bibliographic items which would enable effective retrieval.

- Download a sample of records from various electronic databases available at the UCE School of Information Studies. (I had considered evaluating paper thesauri for the databases: however, enquiries to producers revealed that such thesauri were either no longer produced on paper or would not be obtainable).

- Test the subject indexing of the sample records against the criteria drawn up and analyse the results.

- Drawing on the information gained, devise a framework for more effective subject indexing and apply this to a small sample of records from an existing database as a demonstration of the relevance of the indexing criteria adopted.

\section{Literature review}

In the first stage of research, I carried out a comprehensive review of existing literature over several months - journals, conference papers, monographs and dissertations, and relevant Web pages such as BUBL.

Previous research had uncovered a number of key points. Past design of electronic bibliographic databases was shaped by the information needs of scientists and engineers rather than of humanists; the two domains have quite different needs and practices (1). In contrast to the sciences, there was very little research into the information needs of humanities scholars before the 1980s. Some information professionals therefore assumed that humanities research and electronic search tools were inherently incompatible.

Several key studies of terminology and indexing issues were carried out in the 1980s (2). These largely concurred in the importance of several key factors in humanities subject indexing: names, time periods, geographical areas, and interpretative stance - which were later used in devising my criteria for analysis and my own index.

\section{Database selection}

Key discipline areas of the humanities were identified from the literature review. The disciplines most often cited by researchers as fundamental to the humanities were: art and/or the history of art; literature; history; and philosophy. Religion was also mentioned.

I then identified bibliographic databases covering these key disciplines which were available via DIALOG at the School of Information Studies at UCE. Where more than one database covered a given discipline, the database with the wider subject coverage was preferred, as wider coverage might reveal a broader range of subject indexing issues.

The selected databases were:

- Arts and Humanities Search (hereafter AHS), produced by the Institute for Scientific Information (ISI)

- Historical Abstracts (HisAbs), produced by $\mathrm{ABC}-\mathrm{Clio}$, Inc.

- Philosopher's Index (Phillnd), produced by the Philosophy Documentation Center

- Art Literature International (RILA), produced by RILA, the International Repertory of the Literature of Art.

There were two further benefits in using this selection for analysis. First, each database comes from a different producer, so that a range of indexing practices could be investigated. Second, this selection would enable the comparison of three discipline-specific databases with a large cross-disciplinary database (AHS). 
Framing the Subject

Hazel Rothera

\section{Selection of bibliographic records for analysis}

The purpose of the planned analysis was to survey current indexing practice in several major humanities databases. A straightforward analysis of the ' $n$ ' most recent records in each database could introduce bias - for example, an uneven distribution of topics, languages or publishers being indexed. I therefore decided to take a random sample from a collection of recent records. As DIALOG publishes figures relating to updates, a reasonable estimate can be made of the "relative newness" of particular records or groups of records.

Taking the equivalent of one week's update for each database, whether or not the updates actually occurred every seven days, gave a target subset of 2813 records. Reference to Krejcie and Morgan's "Table for determining sample size from a given population" (3) gave a sample size of 338 from the 2,813 and the sample taken from each database was:

\section{AHS 240 records HisAbs 60 records}

RILA 23 records Phillnd 15 records

Records were selected using Rand Corporation's A Million Random Digits table, as reproduced Powell (4) - to give an independent random sample from each stratum.

\section{Design of framework for analysis}

In order to measure the effectiveness of subject indexing in the database samples under analysis, I proposed to examine the words and phrases occurring in the descriptor fields of the bibliographic records, and to determine how many of the essential and desirable subject indexing criteria were fulfilled in each record. The methodology proposed for analysis of the bibliographic records' subject descriptor fields was to be a form of content analysis, in which categories or sets of codes are used to transform content data into quantifiable.

A framework was drawn up, using an Excel spreadsheet, to carry out the content analysis of bibliographic records and to arrive at a "score" for each record's fulfilment of the indexing criteria. Previous research suggested that names, chronological indicators, geographical indicators and discipline terms were essential indexing elements in all the broad discipline areas of the databases selected. The other criteria, such as historical event, scholarly approach, or genre, were desirable but not essential. I therefore decided to weight the categories in the analytical framework accordingly, assigning a column in the analytical spreadsheet to each.

CRITERION Points per occurrence

$\begin{array}{ll}\text { Name (personal, group, institution) } & 2 \\ \text { Geographic indicator } & 2 \\ \text { Chronological indicator } & 2 \\ \text { Discipline indicator } & 2 \\ \text { Influence indicator } & 1 \\ \text { Scholarly approach indicator } & 1 \\ \text { Theme indicator } & 1 \\ \text { Political entity } & 1 \\ \text { Event } & 1 \\ \text { Language of subject } & 1 \\ \text { Genre of subject } & 1 \\ \text { Medium of subject } & 1 \\ \text { Context of commentating work } & 1\end{array}$

Each element was also assigned a second column in the framework in which a score of 1 would be entered if the element figured in its own separate field.

\section{Analysis of records}

Sample records were printed out and analysed according to the analytical framework described above. Each term (word or phrase) occurring in the descriptor fields of the sample records was coded into one of the categories. The aim of the research was to investigate the indexing of elements describing the subject matter of a document, rather than those elements of "metadata" describing the document itself. 
Fields identifying the author or corporate source of an article, and the journal in which it appeared, were therefore excluded, as they consist of such meta-data, and information they convey about an article's subject is indirect and can be misleading (for example, articles on art may appear in architectural journals). Similarly, the titles of documents in the humanities are often ambiguous, punning or making allusions, rather than straightforwardly describing the subject of the document. (For example: "Through the Shattering Glass : Cervantes and the Self-Made World" by Spadaccini and Talens). Title fields were therefore also excluded as not reliably providing useful subject information. Since author, title and document source fields are routinely provided by bibliographic databases, analysing them would not help to differentiate between well-indexed and poorly-indexed databases.

\section{Devising and demonstrating the subject indexing model}

The final stage of the research involved using the criteria previously identified, and observations from the analysis, to formulate a subject indexing model. This was then applied to a small sample of existing bibliographic records (taken from one of the databases used in the analysis) in order to demonstrate its use and to highlight key challenges and issues involved in subject indexing. The indexing model was a "paper" rather than a "working" model, although it was envisaged as an index designed ultimately for use in electronic rather than paper form.

Devising the model involved:

- $\quad$ selecting subject elements, or concepts, to be indexed;

- devising the different fields in which these elements would be indexed;

- determining rules or guidelines for the form in which to index the various elements;

- deciding where appropriate on controlled vocabularies, and drawing up partial thesauri.
Indexing elements Four key elements of subject searching have consistently been stressed by researchers into humanists' information needs. These are:

- names (of individuals, groups or institutions);

- geographical elements;

- time periods (both specific dates, and more general indicators such as "Middle Ages";

- and the discipline or subject terms which have specific, if sometimes varying, meanings for humanities scholars.

As Langridge pointed out in his 1976 Classification and indexing in the humanities, these elements -"the artist and his work, the individual, the events of history" - consistently define the parameters of humanities research (5). Anita Lowry's 1982 study of quality issues focused on deficiencies in these four elements, which she felt to be key (6). Wiberley's 1983 investigation of indexing terms in humanities reference books stressed the high proportion of precise, proper terms which were used (7).

These findings have been borne out in the 1990s by research into the online searching behaviour of humanities researchers in the Getty Online Searching Project. Analysing natural-language statements of the subjects of searches, Bates et al. found that $45 \%$ of them involved the use of personal names, while geographical terms, chronological terms, and discipline-related terms were also key. I therefore considered these four elements to be essential to effective subject indexing in humanities databases (8).

The above research, and further work by Tibbo (9) and Stebelman (10), yielded further elements which, while not essential, were extremely useful in the indexing of humanities documents. These included:

- geopolitical units;

- historical events; 
- topic- or theme-defining terms;

- genre;

- influence, and

- indicators of scholarly context.

These indicators were also incorporated into my criteria for effective subject indexing.

\section{Assessment of Results}

The results were assembled in an Excel spreadsheet. This enabled analysis and graphical representation of: the overall scores of particular databases; how well different criteria were indexed across different databases; and how well individual databases performed in specific areas.

Overall, the results suggested that current practice in the four major humanities databases analysed fell considerably short of "effective" subject indexing as defined by the criteria described above. There was no maximum possible score, but it was possible to calculate a "good score" which would have been obtained if each record had scored for one entry in each category. On this basis, the four databases overall scored just $31 \%$ of a "good score".

AHS was the lowest-scoring database, with just $16 \%$ of a "good score" across the database. This database provides a good deal of bibliographic detail, but very little subject information; it is very difficult to use effectively unless the searcher is already following up a specific citation. Although DIALOG documentation states that subject descriptors have been available since 1991, only one of the 240 records in the AHS sample contained any of these "Keywords Plus" identifiers. Most ALS records contained no subject indexing at all, providing no indication of people, places, periods or disciplines which the document was about. The few points scored by ALS were picked up due to my decision to include its "Journal Subject" field in the analysis; this gives the discipline into which the journal containing the indexed document falls, although it is not a precise indicator of an individual article's subject or discipline area.
Phillnd scored $27 \%$ of a "good score". However, its indexing was primarily on oneword theme or discipline terms; previous research had indicated that such terms were particularly important to information seekers in philosophy. Some records included very informative abstracts written by the article authors. A separate field was allotted to the indexing of named individuals. Article titles were also much more directly descriptive of content than is often the case in other humanities disciplines.

HisA bs scored $71 \%$ of a "good score". It also provides abstracts. One "Descriptors" field contains all its remaining subject information except chronological indicators, which are detailed and involve enumerating decades and/or centuries to give time periods. This made chronological indicators HisAbs' outstandingly high-scoring feature - with geographical, theme and name terms also indexed.

RILA was the most thoroughly subjectindexed database. It scored $85 \%$ of a "good score" overall, with lengthy and detailed indexing covering more of the essential and desirable indexing criteria than other databases. Discipline indicators, names, geographic and chronological indicators - the essential criteria - were all covered, as were scholarly approach, theme, event, genre, medium, and contextual information.

Key indexing criteria By adjusting the databases' scores to account for the varying proportion of the sample which each database represented, it was possible to compare their performances directly across the key criteria. By these adjusted scores, RILA, the best-indexed database, scored over 5 times as many indexing points as AHS. This reflected my general experience while searching and carrying out analysis.

Of the four essential criteria, names and discipline indicators were most often indexed by RILA, with both RILA and HisAbs scoring well on geographic indicators. Discipline indicators were 
also well-used in Phillnd. Chronological indicators, as noted above, were most often indexed by HisAbs. The poor performance of several databases in the area of proper names (personal and institutional names, titles of works) was particularly striking given the availability in electronic form of standardised union lists for these features, - from the Library of Congress, for example.

As expected, the non-essential criteria were indexed less frequently in all four databases than the essential ones. Theme indicators were most often used, particularly in PhilInd; context indicators were also frequent. Scholarly approach, artistic medium, and genre were indexed by RILA; HisAbs made some use of geopolitical indicators, and AHS occasionally indexed language.

\section{Issues encountered}

Drawing up indexing criteria, and reflecting on the problems of subject indexing, threw up a number of interesting issues. The challenge in producing my own demonstration subject index was to attempt to resolve these in ways which would enable effective subject searching. This thought-process, and its application in my own indexing, was a very enjoyable aspect of the research.

One problem was that of "multi-layered subjects": humanities scholars tend to take previous works either primary works such as plays or paintings, or previous critical writing - as the subjects of their own further work. Thus, who or what is or are the subjects of an article about the Spanish historian, Maravall's interpretation of Don Quixote? I decided it was often necessary to index "layers" of subjects - in this example, to specify Cervantes, Don Quixote, Maravall, and the title of Maravall's work as subjects.

Forms of names also had to be fixed (which in a real database could be overcome by using a preexisting controlled index). Geographical areas were similarly defined - for example "Europe/Western Europe/Spain". I also devised chronological indicators, which combined specification of relevant decades or centuries with more general "period indicators" such as "Spanish Golden Age" or "Middle Ages". These, while open to interpretation, are valuable to humanities researchers, as artistic schools and literary movements rarely correspond neatly to decades or centuries.

Where discipline indicators were concerned, I decided to make broad definitions ("history", "literature"), as these can be very useful to humanities researchers when combined with other indicators such as the chronological or geographic. A controlled vocabulary was used for this field, and a non-controlled one for a further "themes" field to enable more specific topic terms to be assigned; these could either be picked up from the work or its abstract, or created by the indexer.

The most interesting non-essential fields to index were scholarly approach, and influence. Scholarly approach, while a key aspect of humanities studies, is very difficult to assign; I used literary-critical terms from various reference works, but in a real database it might be preferable to ask article authors to assign their own "labels" from a controlled list. As for influence, I felt it important to index two aspects very frequently the subject of discussion in the humanities: the influences of previous works on a subject work, and the influence that work has had on subsequent works/authors. I therefore indexed these two concepts in two separate fields.

\section{Demonstration}

Having considered these issues and drawn up an indexing framework to incorporate all the criteria, I applied my framework to a selection of records. The subject of these records was the life and work of the 16 th-17th century author Miguel de Cervantes - in particular his most famous novel, Don Quixote. This was an area in which I personally had detailed subject knowledge and therefore felt able to address key subject indexing problems and issues. (As it transpired, getting to grips with the challenges of providing concise but effective subject indexing was one of the most enjoyable and fulfilling aspects of the research!) 
The sample had to be small enough to be adequately indexed within the time constraints of the research, while providing a broad enough range of issues to demonstrate various features of the indexing model. So a set of 25 records relating to Don Quixote was downloaded from the Historical Abstracts database.
In the dissertation, each record was presented with my subject indexing following the original bibliographic record and its original subject indexing in Historical Abstracts . My indexing was intended to replace the DESCRIPTORS and HISTORICAL PERIOD fields of the original. Two examples follow, with my indexing categories in bold:

Original record:

The value of money in Cervantes's time

SOBRE EL VALOR DE LA MONEDA EN LA EPOCA DE CERVANTES

Palacin Iglesias, Gregorio

Humanitas (Mexico) 1972 13: 599-612

DOCUMENT TYPE: Article

ABSTRACT: analyses the usage of money in Miguel de Cervantes's Don Quixote to determine the value of money in early 16th-century Spain.

DESCRIPTORS: Cervantes, Miguel de (Don Quixote); 1570s-1610s; Money, value of; Spain

HISTORICAL PERIOD: 1570D 1580D 1590D 1600D 1610D 1600H

HISTORICAL PERIOD (Starting): 1570s

HISTORICAL PERIOD (Ending): 1610s

My subject indexing :

$\begin{array}{ll}\text { Names: } & \begin{array}{l}\text { Cervantes Saavedra, Miguel de, Spanish, novelist, 1547- } \\ 1616\end{array} \\ \text { Works: } & \begin{array}{l}\text { El ingenioso hidalgo Don Quijote de la Mancha (1605, } \\ 1615)\end{array} \\ \text { Geographic area: } & \text { Europe; Western Europe; Iberia; Spain } \\ \text { Political entity: } & \begin{array}{l}\text { Habsburg Monarchy; Felipe II (1556-1598), Felipe III } \\ (1598-1621)\end{array} \\ \text { Chronology: } & 1500 \mathrm{C} ; 1570 \mathrm{D}, 1580 \mathrm{D}, 1590 \mathrm{D}: 1600 \mathrm{C} ; 1600 \mathrm{D}, 1610 \mathrm{D} \text { : } \\ & 1605 \mathrm{Y}, 1615 \mathrm{Y} \\ \text { Discipline: } & \text { literature: economics: history } \\ \text { Scholarly approach: } & \text { historical criticism } \\ \text { Themes: } & \text { money: economics } \\ \text { Language: } & \text { Spanish } \\ \text { Genre: } & \text { novel } \\ \text { Medium: } & \text { fiction }\end{array}$


Original record:

A Quixotic motive in works by Vladislav Vancura

DONKICHOTSKY MOTIV V DILE VLADISLAVA VANCURY

Holy, Jiri

Literarni Arch (Czechoslovakia) 1982 13-15: 37-50

DOCUMENT TYPE: Article

LANGUAGE(S): Czech

ABSTRACT: A typological and genetical comparison between Don Quixote by

Cervantes and the first novel by Vancura, The Baker Jan Marhoul, and also Citizen Don Quixote.

DESCRIPTORS: Novels: Cervantes, Miguel de (Don Quixote); 1924-1937; Vancura, Vladislav; Czechoslovakia

HISTORICAL PERIOD: 1920D 1930D 1900H

HISTORICAL PERIOD (Starting): 1924

HISTORICAL PERIOD (Ending): 1937

My subject indexing :

\begin{tabular}{|ll|}
\hline Names: & Cervantes Saavedra, Miguel de, Spanish, novelist, 1547- \\
& 1616 \\
Works: & El ingenioso hidalgo Don Quijote de la Mancha (1605, \\
& $1615)$ \\
Geographic area: & Europe; Western Europe; Iberia; Spain: Eastern Europe; \\
& Czechoslovakia \\
Chronology: & 1600C; 1600D, 1610D; 1605Y, 1615Y: 1900C; 1920D, \\
& $1930 \mathrm{D} ; 1924 \mathrm{Y}, 1937 \mathrm{Y}$ \\
Discipline: & literature \\
Scholarly approach: & Comparative criticism \\
Influence of: & Cervantes Saavedra, Miguel de, Spanish, novelist, 1547- \\
& 1616: El \\
Influence on: & ingenioso hidalgo Don Quijote de la Mancha (1605, 1615) \\
& Vancura, Vladislav: Baker Jan Marhoul (1924): Citizen Don \\
Language: & $\quad$ Quixote (1937) \\
Medium: & Spanish: Czech \\
& fiction
\end{tabular}




\section{Conclusions}

In summing up my research, I concluded that the information needs of humanities scholars have often been neglected by the providers of electronic databases. This may be due in part to the history of electronic database provision, which was initially based on scientific information paradigms. Effective subject indexing in humanities disciplines is also made complex and difficult by a number of specific factors; for example, their complex multiple subjects. The subtle, rich and highly nuanced language which is a highly-prized part of humanities scholarship makes term indexing both essential and extremely difficult.

However, previous research also demonstrated that there were many very concrete concepts used by humanists to define the parameters of their studies which could usefully be employed to make subject indexing effective. The aim of the indexing analysis I carried out was to find out whether key humanities databases from a number of producers, made available by one of the major online hosts, DIALOG, were currently making effective use of these concepts. Overall the indexing fulfilled the key criteria only to a limited degree. This result demonstrated the potential for more detailed subject indexing models based on the essential and desirable subject criteria.

\section{Recommendations for future research}

This research demonstrated that it is possible to apply criteria for effective subject indexing, based on previous research into the information needs of humanists, to a sample of existing records from a bibliographic database. A number of possibilities exist for future research in this area and for future applications of such indexing models.

I made only limited application of the indexing model which was developed. Future research might examine the model's application to different subject areas. Alternatively, working models might be constructed, either in paper or in electronic format, to test the model's relevance and ease of use by applying "real" search queries to it. Such models could also test the hypothesis proposed by several researchers that, if provided with databases that met their needs, humanists would be no less eager to use them than other academic groups.

There is also scope for further research into the current indexing practice and policy of major database producers in the humanities. Research might, for example, examine the level of detail and depth achieved by current subject indexing, or investigate the accuracy and consistency with which terms were applied, neither of which I was able to address.

\section{Future developments}

I did not attempt to investigate in any depth the current attitude of major database suppliers and hosts to the provision of effective humanities services, although it was noted that DIALOG, for example, provides relatively few humanities databases in comparison to its science and business services. Some commentators have argued that humanities databases are not commercially attractive.

Providing subject indexing for records of humanities documents can be a complex and time-consuming process due to the nature of humanities scholarship and the difficulty of assigning descriptive terms consistently. Given the current state of automatic natural-language indexing, it is difficult to envisage such indexing being carried out other than by a human subject expert. However, I also found that devising a carefully structured indexing model, with clearly defined fields which make use of the many very concrete concepts also used by humanists (names, geography and chronology for example), made subject indexing a faster, more straightforward and more consistent process.

The production of databases which could be searched effectively and quickly with relevant results might do much to raise the profile of electronic databases within the humanities, and correspondingly increase their popularity and commercial viability. Indexing models based on the criteria proposed need not necessarily be applied exclusively in the context of "traditional" online databases. Effective subject indexing of bibliographic records could be applied in many other contexts, such as in small-scale CD-ROM subject bibliographies or on the World Wide Web. 
Recent developments in the search engines and search interfaces of bibliographic databases could also be applied to humanities databases indexed in this way. "Term mapping", for example, which automatically searches on a controlled index term when a synonym for it is entered, could make searching easier and more effective for humanists and information specialists, as could pull-down indexes to encourage searchers to select terms from controlled lists.

I concluded that such improvements to humanities databases could both increase the market for electronic bibliographic services in academia, and enhance the work of scholars by making the enormous and ever-increasing corpus of existing published work more quickly, easily and selectively available to them.

\section{Final Thoughts}

Revisiting the dissertation research carried out nearly two years ago to produce this article has been a thought-provoking experience, reminding me how pressured I often felt by time and by the minutiae of presenting the dissertation in its final, hopefully fully-referenced and error-free form. I was aware of the limited scope of this research and that there were areas, such as the feasibility in practice of producing such detailed subject indexing for commercial databases, which I was unable to explore.

Having left library school and almost immediately moved into employment in a different sector of the library world, I am now aware that my.ability to follow developments in the area of my research has been limited, and that some of my conclusions may well have been overtaken by events. I also know that like myself a number of my fellowstudents, who would have liked to disseminate the hard-won findings of their research more widely, have found it difficult or impossible to combine full-time work in their first professional posts with further re-writing of dissertations for publication. So much research is carried out in the LIS field every year by Masters students that all ideas for encouraging publication, and for making it easier for hard-pressed entrants to the profession to present their research findings, must surely be welcome.

\section{References}

(I) Nicholas, David. Review of: Stone, S., Humanities information research, 1980, and Corkhill, C., et al, Doctoral students in humanities. Journal of Documentation 38 (2), 1982, pp. I39-1 40.

Tibbo, Helen R. Indexing for the humanities. Journal of the American Society for Information Science 45 (8), 1994, pp. 607-619.

Wiberley, Stephen E. Subject access in the humanities and the precision of the humanist's vocabulary. Library Quarterly 53, 1983, pp. 420-433.

Wiberley, Stephen E. Names in space and time: the indexing vocabulary of the humanities. Library Quarterly 58 (1), 1988, pp. 1-28.

(2) Bates, M.J.The design of databases and other information resources for humanities scholars: the Getty online searching project report no. 4. Online and CD-ROM Review I8 (6), 1994, pp. 331-40.

Bates, M.J., et al...An analysis of search terminology used by humanities scholars: the Getty online searching project report no. I. Library and Information Science Research 17 (1), 1995, pp. 5-40.

Everett, David, and Pilachowski, D.M.What's in a name? Looking for people online - humanities. Database 9 (5), 1986, pp. 26-34.

Lowry, Anita.A consumer's report on humanities databases. Technicalities 2 (8), 1982, pp. I-3, | I- 12.

Slatta, Richard W. Telecommunication for the humanities and social sciences. Microcomputers for Information Management 3 (2), 1986, pp. 91 = 110.

Walker, Geraldene, and Atkinson, Steven D. Information access in the humanities: perils and pitfalls. Library HiTech 9 (1), 1991, pp. 23-34.

Wiberley, 1983, 1998, op. cit.

(3) Powell, Ronald R. Basic research methods for librarians. 2nd rev. ed. U.S.A:Ablex, 1991. (Information management, policies and services series), p. 75 .

(4) Ibid., pp. 66-68.

(5) Langridge, D.W. Classification and indexing in the humanities. London: Butterworths, 1976.

(6) Lowry, 1982, op. cit.

(7) Wiberley, 1983, op. cit.

(8) Bates et al, 1994, 1995, op. cit.

(9) Tibbo, 1994, op. cit.

(10) Stebelman, Scott S.Vocabulary control and the humanities: a case study of the MLA International Bibliography. Reference Librarian 47 (1), 1994, pp. $61-78$. 